ANNALES

POLONICI MATHEMATICI

$95.3(2009)$

\title{
Lifting right-invariant vector fields and prolongation of connections
}

\author{
by W. M. Mikulski (Kraków)
}

\begin{abstract}
We describe all $\mathcal{P} \mathcal{B}_{m}(G)$-gauge-natural operators $\mathcal{A}$ lifting right-invariant vector fields $X$ on principal $G$-bundles $P \rightarrow M$ with $m$-dimensional bases into vector fields $\mathcal{A}(X)$ on the $r$ th order principal prolongation $W^{r} P=P^{r} M \times_{M} J^{r} P$ of $P \rightarrow M$. In other words, we classify all $\mathcal{P} \mathcal{B}_{m}(G)$-natural transformations $J^{r} L P \times_{M} W^{r} P \rightarrow T W^{r} P=$ $L W^{r} P \times_{M} W^{r} P$ covering the identity of $W^{r} P$, where $J^{r} L P$ is the $r$-jet prolongation of the Lie algebroid $L P=T P / G$ of $P$, i.e. we find all $\mathcal{P B}_{m}(G)$-natural transformations which are similar to the Kumpera-Spencer isomorphism $J^{r} L P=L W^{r} P$. We formulate axioms which characterize the flow operator of the gauge-bundle $W^{r} P \rightarrow M$. We apply the flow operator to prolongations of connections.
\end{abstract}

0. Introduction. Let $G$ be a Lie group with Lie algebra $\mathcal{L}(G)$ and $e \in G$ be the unit element. Let $\mathcal{P B}_{m}(G)$ denote the category of all principal $G$-bundles with $m$-dimensional bases and their (local) principal bundle isomorphisms over the identity group homomorphism.

Let $\Phi, \Psi: P \rightarrow Q$ be $\mathcal{P} \mathcal{B}_{m}(G)$-maps. Let $x \in M$. The following conditions are equivalent: (i) $j_{p_{0}}^{r} \Phi=j_{p_{0}}^{r} \Psi$ for some $p_{0} \in P_{x}$; (ii) $j_{p}^{r} \Phi=j_{p}^{r} \Psi$ for any $p \in P_{x}$. We write $j_{x}^{r} \Phi=j_{x}^{r} \Psi$ if these conditions are satisfied (see [3]).

Let $P \rightarrow M$ be a principal $G$-bundle with $m$-dimensional basis. Its $r$ th principal prolongation $W^{r} P$ is defined as the space of all $r$-jets $j_{0}^{r} \varphi$ of local trivializations $\varphi: \mathbb{R}^{m} \times G \rightarrow P$. By [3], $W^{r} P \rightarrow M$ is a principal bundle with the structure group $W_{m}^{r} G:=J_{0}^{r}\left(\mathbb{R}^{m} \times G, \mathbb{R}^{m} \times G\right)_{0}$, and the fibred manifold $W^{r} P \rightarrow M$ coincides with the fibred product $P^{r} M \times_{M} J^{r} P$, where $P^{r} M=\operatorname{inv} J_{0}^{r}\left(\mathbb{R}^{m}, M\right)$ is the $r$ th order frame bundle of $M$. Moreover, $W_{m}^{r} G=G_{m}^{r} \rtimes T_{m}^{r} G$, and $\operatorname{dim}\left(W_{m}^{r} G\right)=m\left(C_{r}^{m+r}-1\right)+\operatorname{dim}(G) C_{r}^{m+r}$, where $C_{k}^{n}=n ! / k !(n-k)$ !. Every $\mathcal{P} \mathcal{B}_{m}(G)$-map $\Phi: P \rightarrow Q$ can be extended (via composition of jets) to a principal bundle (local) isomorphism $W^{r} \Phi$ :

2000 Mathematics Subject Classification: 58A20, 58A32.

Key words and phrases: principal bundle, higher order principal prolongation, rightinvariant vector field, principal connection, higher order linear connection, Lie algebroid, natural operator. 
$W^{r} P \rightarrow W^{r} Q$. Thus we obtain a gauge-bundle functor $W^{r}: \mathcal{P} \mathcal{B}_{m}(G) \rightarrow$ $\mathcal{P} \mathcal{B}_{m}\left(W_{m}^{r} G\right)$ in the sense of [3]. This functor plays a central role in the theory of gauge-natural bundles and gauge-natural operators because any gauge-natural bundle functor $F: \mathcal{P} \mathcal{B}_{m}(G) \rightarrow \mathcal{F M}$ of order $r$ is associated to $W^{r}$ (see [3]).

Another example of a gauge-bundle functor on $\mathcal{P} \mathcal{B}_{m}(G)$ is the functor $L$ associating to any $\mathcal{P B}_{m}(G)$-object $P \rightarrow M$ the Lie algebroid $L P=T P / G$ of $P$ and to any $\mathcal{P B}_{m}(G)$-map $\Phi: P \rightarrow Q$ the induced Lie algebroid map $L(\Phi): L P \rightarrow L Q$. Lie algebroids are a modern tool in several branches of mathematical physics. Smooth sections of $L P \rightarrow M$ are in bijection with right-invariant vector fields on $P$.

A classical result of Kumpera and Spencer [4] says that there is an isomorphism (identification) $J^{r} L P=L W^{r} P$ between the $r$-jet prolongation $J^{r} L P$ of $L P \rightarrow M$ and the Lie algebroid $L W^{r} P$ of $W^{r} P \rightarrow M$ (see also [2]). In the present note (see Remark 1), using the gauge-natural operator technique we classify all $\mathcal{P} \mathcal{B}_{m}(G)$-natural transformations $J^{r} L P \times_{M} W^{r} P \rightarrow T W^{r} P=$ $L W^{r} P \times_{M} W^{r} P$ covering the identity of $W^{r} P$, i.e. we find all $\mathcal{P} \mathcal{B}_{m}(G)$-natural transformations which are similar to the Kumpera-Spencer isomorphism.

Roughly speaking, a gauge-natural operator is a canonical construction of a gauge geometric object (a section of a gauge bundle) from some given gauge geometric objects. Very important gauge-natural objects are principal connections on principal bundles. So, constructions of principal connections from other principal ones are especially important. To obtain such constructions, some vector fields obtained from right-invariant vector fields are useful (see e.g. [7] in the case of the trivial Lie group $G=\{e\}$ ). Taking into account the above remarks, we see that a complete classification of all $\mathcal{P} \mathcal{B}_{m}(G)$-gauge-natural operators $\mathcal{A}: T_{G \text {-inv }} \rightsquigarrow T W^{r}$ in the sense of Definition 1 is especially important.

Definition 1 ([3]). A $\mathcal{P} \mathcal{B}_{m}(G)$-gauge-natural operator $\mathcal{A}: T_{G \text {-inv }} \rightsquigarrow$ $T W^{r}$ is a family of $\mathcal{P} \mathcal{B}_{m}(G)$-invariant regular operators (functions)

$$
\mathcal{A}=\mathcal{A}_{P}: \mathcal{X}_{G \text {-inv }}(P) \rightarrow \mathcal{X}\left(W^{r} P\right)
$$

from the set $\mathcal{X}_{G \text {-inv }}(P)$ of all right-invariant vector fields on $P$ (sections of the Lie algebroid $L P$ of $P$ ) into the set $\mathcal{X}\left(W^{r} P\right)$ of all vector fields on $W^{r} P$ for any $\mathcal{P B}_{m}(G)$-object $P \rightarrow M$. The invariance means that if $X_{1} \in \mathcal{X}_{G \text {-inv }}(P)$ and $X_{2} \in \mathcal{X}_{G \text {-inv }}(Q)$ are related by a $\mathcal{P} \mathcal{B}_{m}(G)$-map $\Phi: P \rightarrow Q$ then $\mathcal{A}_{P}\left(X_{1}\right)$ and $\mathcal{A}_{Q}\left(X_{2}\right)$ are related by $W^{r} \Phi$. The regularity means that $\mathcal{A}$ transforms smoothly parametrized families of right-invariant vector fields into smoothly parametrized families of vector fields.

A $\mathcal{P} \mathcal{B}_{m}(G)$-gauge-natural operator $\mathcal{A}: T_{G \text {-inv }} \rightsquigarrow T W^{r}$ is said to be of vertical type if $\mathcal{A}_{P}(X)$ is a vertical vector field on $W^{r} P \rightarrow M$ for any right-invariant vector field $X$ on an arbitrary $\mathcal{P} \mathcal{B}_{m}(G)$-object $P \rightarrow M$. 
Let $k$ be a non-negative integer. A $\mathcal{P} \mathcal{B}_{m}(G)$-gauge-natural operator $\mathcal{A}$ : $T_{G \text {-inv }} \rightsquigarrow T W^{r}$ is said to be of order $\leq k$ if for any right-invariant vector fields $X_{1}$ and $X_{2}$ on $P \rightarrow M$ and $x \in M$ the equality of $k$-jets $j_{x}^{k}\left(X_{1}\right)=j_{x}^{k}\left(X_{2}\right)$ implies $\mathcal{A}_{P}\left(X_{1}\right)=\mathcal{A}_{P}\left(X_{2}\right)$ on the fibre $\left(W^{r} P\right)_{x}$.

EXAMPLE 1. An example of a $\mathcal{P} \mathcal{B}_{m}(G)$-gauge-natural operator $\mathcal{A}: T_{G \text {-inv }}$ $\rightsquigarrow T W^{r}$ of order $\leq r$ is the flow operator $\mathcal{W}^{r}$ sending a right-invariant vector field $X$ on a $\mathcal{P B}_{m}(G)$-object $P \rightarrow M$ into the complete lift $\mathcal{W}^{r} X$ of $X$ to $W^{r} P$. We recall that $\mathcal{W}^{r} X$ is the vector field on $W^{r} P$ such that if $\left\{\Phi_{t}\right\}$ is the flow of $X$ then $\left\{W^{r} \Phi_{t}\right\}$ is the flow of $\mathcal{W}^{r} X$. (We observe that $X$ is a right-invariant vector field on a $\mathcal{P} \mathcal{B}_{m}(G)$-object iff its flow is formed by local $\mathcal{P} \mathcal{B}_{m}(G)$-maps. That is why we can apply the functor $W^{r}: \mathcal{P} \mathcal{B}_{m}(G) \rightarrow$ $\mathcal{P} \mathcal{B}_{m}\left(W_{m}^{r} G\right)$ to the flow of $X$.)

ExAmple 2. Let $E \in \mathcal{L}\left(W_{m}^{r} G\right)$. Let $E^{*}$ denote the fundamental vector field on $W^{r} P$ corresponding to $E$ for any $\mathcal{P B}_{m}(G)$-object $P \rightarrow M$. We have the (constant) $\mathcal{P} \mathcal{B}_{m}(G)$-gauge-natural operator $E^{*}: T_{G \text {-inv }} \rightsquigarrow T W^{r}$ defined by $\left(E^{*}\right)_{P}(X)=E^{*}$ for any right-invariant vector field $X$ on $P$. Clearly, the operator $E^{*}$ is of vertical type.

In the present article we solve the following two problems.

Problem 1. Classify all $\mathcal{P} \mathcal{B}_{m}(G)$-gauge-natural operators $\mathcal{A}: T_{G \text {-inv }} \rightsquigarrow$ $T W^{r}$.

Problem 2. Find axioms which characterize the flow operator $\mathcal{W}^{r}$.

The solution of Problem 1 is given in Theorem 1. We prove that the set of all $\mathcal{P} \mathcal{B}_{m}(G)$-gauge-natural operators $\mathcal{A}: T_{G \text {-inv }} \rightsquigarrow T W^{r}$ is a free finite-dimensional module over an algebra. We will construct a basis of this module explicitly. To prove Theorem 1 we modify the proof of the main result in [5]. The solution of Problem 2 is given in Theorem 2. It seems that the characterization of the flow operator $\mathcal{W}^{r}$ may be useful because the operator plays a central role in the theory of gauge bundle functors (as it is defined for any gauge bundle functor and has some good algebraic properties).

Constructions on vector fields have been studied in many papers (see e.g. [1], [3], [5], [7]). Some of them were applied in constructions on connections (see e.g. [3], [7]). In Section 8, using the flow operator $\mathcal{W}^{r}$ we construct a principal connection $\widetilde{\mathcal{W}}^{r}(\Gamma, \Lambda)$ on the principal bundle $W^{r} P \rightarrow P$.

All manifolds and maps are assumed to be smooth (of class $\mathcal{C}^{\infty}$ ).

\section{Preliminaries}

Lemma 1. Let $X, Y \in \mathcal{X}_{G \text {-inv }}(P)$ be right-invariant vector fields on $p$ : $P \rightarrow M$ and $x \in M$ be a point. Suppose that $j_{x}^{r} X=j_{x}^{r} Y$ and $X$ is not vertical 
over $x$. Then there exists a (locally defined) $\mathcal{P B}_{m}(G)$-map $\Phi: P \rightarrow P$ such that $j_{x}^{r+1}(\Phi)=j_{x}^{r+1}\left(\operatorname{id}_{P}\right)$ and $\Phi_{*} X=Y$ near $x$.

Proof. A direct modification of the proof of Lemma 42.4 in [3].

Proposition 1. Any $\mathcal{P B}_{m}(G)$-gauge-natural operator $\mathcal{A}: T_{G \text {-inv }} \rightsquigarrow$ $T W^{r}$ is of order $\leq r$.

Proof. A replica of the proof of Proposition 42.5 in [3]. We use Lemma 1 instead of Lemma 42.4 in [3].

The Kumpera-Spencer isomorphism $J^{r} L P=L W^{r} P$ yields $J^{r} L P \times_{M}$ $W^{r} P=L W^{r} P \times_{M} W^{r} P=T W^{r} P$. Therefore we have the following lemma.

Lemma 2. Any vector $v \in T_{w} W^{r} P$ with $w \in\left(W^{r} P\right)_{x}$ and $x \in M$ is of the form $\mathcal{W}^{r} X_{w}$ for some $X \in \mathcal{X}_{G \text {-inv }}(P)$. Moreover, $j_{x}^{r} X$ is uniquely determined.

2. The gauge-natural operators $\mathcal{B}: T_{G \text {-inv }} \rightsquigarrow T^{(0,0)} W^{r}$. If in Definition 1 we replace $\mathcal{X}\left(W^{r} P\right)$ by the space $C^{\infty}\left(W^{r} P\right)$ of mappings $W^{r} P \rightarrow \mathbb{R}$, we obtain the concept of a $\mathcal{P} \mathcal{B}_{m}(G)$-gauge-natural operator $\mathcal{B}: T_{G \text {-inv }} \rightsquigarrow$ $T^{(0,0)} W^{r}$ lifting right-invariant vector fields on $P$ to maps $W^{r} P \rightarrow \mathbb{R}$.

EXAMPLE 3. We have the following general example of $\mathcal{P} \mathcal{B}_{m}(G)$-gaugenatural operators $T_{G \text {-inv }} \rightsquigarrow T^{(0,0)} W^{r}$. Let $\lambda: J_{0}^{r-1}\left(T_{G \text {-inv }}\left(\mathbb{R}^{m} \times G\right)\right) \rightarrow \mathbb{R}$ be a map, where $J_{0}^{r-1}\left(T_{G \text {-inv }}\left(\mathbb{R}^{m} \times G\right)\right)$ is the vector space of all $(r-1)$-jets $j_{0}^{r-1} X$ at $0 \in \mathbb{R}^{m}$ of right-invariant vector fields $X \in \mathcal{X}_{G \text {-inv }}\left(\mathbb{R}^{m} \times G\right)$. Then given a right-invariant vector field $X$ on a $\mathcal{P B}_{m}(G)$-object $P \rightarrow M$ we have $\mathcal{B}^{\langle\lambda\rangle}(X): W^{r} P \rightarrow \mathbb{R}$ given by

$$
\mathcal{B}^{\langle\lambda\rangle}(X)\left(j_{0}^{r} \Phi\right)=\lambda\left(j_{0}^{r-1}\left(\Phi_{*}^{-1} X\right)\right)
$$

for all $j_{0}^{r} \Phi \in\left(W^{r} P\right)_{x}, x \in M$, where $\Phi: \mathbb{R}^{m} \times G \rightarrow P$ is a $\mathcal{P} \mathcal{B}_{m}(G)$-map with the underlying map $\varphi: \mathbb{R}^{m} \rightarrow M$ with $\varphi(0)=x$. The correspondence $\mathcal{B}^{\langle\lambda\rangle}$ : $T_{G \text {-inv }} \rightsquigarrow T^{(0,0)} W^{r}$ is a $\mathcal{P} \mathcal{B}_{m}(G)$-gauge-natural operator of order $\leq r-1$ transforming right-invariant vector fields on $P \rightarrow M$ to maps $W^{r} P \rightarrow \mathbb{R}$.

Given $\mathcal{P} \mathcal{B}_{m}(G)$-gauge-natural operators $\mathcal{B}_{1}, \mathcal{B}_{2}: T_{G \text {-inv }} \rightsquigarrow T^{(0,0)} W^{r}$ we have the $\mathcal{P B}_{m}(G)$-gauge-natural operator $\mathcal{B}_{1} \mathcal{B}_{2}: T_{G \text {-inv }} \rightsquigarrow T^{(0,0)} W^{r}$ given by

$$
\left(\mathcal{B}_{1} \mathcal{B}_{2}\right)_{P}(X)=\left(\mathcal{B}_{1}\right)_{P}(X)\left(\mathcal{B}_{2}\right)_{P}(X)
$$

for any right-invariant vector field $X$ on a $\mathcal{P} \mathcal{B}_{m}(G)$-object $P \rightarrow M$. Similarly we define the $\operatorname{sum} \mathcal{B}_{1}+\mathcal{B}_{2}: T_{G \text {-inv }} \rightsquigarrow T^{(0,0)} W^{r}$.

Proposition 2. The map $\lambda \mapsto \mathcal{B}^{\langle\lambda\rangle}$ is an algebra isomorphism from the algebra of smooth maps $J_{0}^{r-1}\left(T_{G \text {-inv }}\left(\mathbb{R}^{m} \times G\right)\right) \rightarrow \mathbb{R}$ onto the algebra of all $\mathcal{P B}_{m}(G)$-gauge-natural operators $T_{G \text {-inv }} \rightsquigarrow T^{(0,0)} W^{r}$.

Proof. Clearly, the map in question is an algebra monomorphism. 
Any $\mathcal{P} \mathcal{B}_{m}(G)$-gauge-natural operator $\mathcal{B}: T_{G \text {-inv }} \rightsquigarrow T^{(0,0)} W^{r}$ of order $\leq r-1$ defines $\lambda: J_{0}^{r-1}\left(T_{G \text {-inv }}\left(\mathbb{R}^{m} \times G\right)\right) \rightarrow \mathbb{R}$ by

$$
\lambda\left(j_{0}^{r-1} X\right)=\mathcal{B}(X)_{j_{0}^{r}\left(\operatorname{id}_{\mathbb{R}^{m} \times G}\right)} .
$$

By an order argument $\lambda$ is well-defined. It is smooth because of the regularity of $\mathcal{B}$ (standard argument using the Boman theorem, see [3]). Then by invariance with respect to local trivialization one can easily see that $\mathcal{B}=\mathcal{B}^{\langle\lambda\rangle}$.

Quite similarly to Proposition 1 , one can show that any $\mathcal{B}$ as above is of order $\leq r-1$.

Thus the map $\lambda \mapsto \mathcal{B}^{\langle\lambda\rangle}$ is an isomorphism.

3. Gauge-natural operators $\mathcal{A}: T_{G \text {-inv }} \rightsquigarrow T W^{r}$ of vertical type. Let $E_{\nu} \in \mathcal{L}\left(W_{m}^{r} G\right)\left(\nu=1, \ldots, \operatorname{dim}\left(W_{m}^{r} G\right)\right)$ be a basis of $\mathcal{L}\left(W_{m}^{r} G\right)$. Then the fundamental vector fields $\left(E_{\nu}\right)^{*}$ for $\nu=1, \ldots, \operatorname{dim}\left(W_{m}^{r} G\right)$ form a basis over $C^{\infty}\left(W^{r} P\right)$ of the module of vertical vector fields on $W^{r} P \rightarrow M$ for any $\mathcal{P B}_{m}(G)$-object $P \rightarrow M$.

The space of all $\mathcal{P} \mathcal{B}_{m}(G)$-gauge-natural operators $T_{G \text {-inv }} \rightsquigarrow T W^{r}$ transforming right-invariant vector fields on $\mathcal{P B}_{m}(G)$-objects $P \rightarrow M$ into vector fields on $W^{r} P$ is a module over the algebra of $\mathcal{P} \mathcal{B}_{m}(G)$-gauge-natural operators $T_{G \text {-inv }} \rightsquigarrow T^{(0,0)} W^{r}$. (Actually, given $\mathcal{P} \mathcal{B}_{m}(G)$-gauge-natural operators $\mathcal{A}: T_{G \text {-inv }} \rightsquigarrow T W^{r}$ and $\mathcal{B}: T_{G \text {-inv }} \rightsquigarrow T^{(0,0)} W^{r}$ we have the $\mathcal{P} \mathcal{B}_{m}(G)$-gaugenatural operator $\mathcal{B A}: T_{G \text {-inv }} \rightsquigarrow T W^{r}$ given by

$$
(\mathcal{B A})_{P}(X)=\mathcal{B}_{P}(X) \mathcal{A}_{P}(X)
$$

for any right-invariant vector field $X$ on a $\mathcal{P B}_{m}(G)$-object $P \rightarrow M$.) Then (by Proposition 1) the above space of operators is a module over the algebra of all maps $J_{0}^{r-1}\left(T_{G \text {-inv }}\left(\mathbb{R}^{m} \times G\right)\right) \rightarrow \mathbb{R}$.

Proposition 3. The (sub)module of all vertical type $\mathcal{P B}_{m}(G)$-gaugenatural operators $\mathcal{A}: T_{G \text {-inv }} \rightsquigarrow T W^{r}$ is free. The operators $\left(E_{\nu}\right)^{*}$ form a basis of this module over $\mathcal{C}^{\infty}\left(J_{0}^{r-1}\left(T_{G \text {-inv }}\left(\mathbb{R}^{m} \times G\right)\right)\right.$.

Proof. Since the fundamental vector fields $\left(E_{\nu}\right)^{*}$ on $W^{r} P$ form a basis of the module of vertical vector fields on $W^{r} P$, any $\mathcal{P} \mathcal{B}_{m}(G)$-gauge-natural operator $\mathcal{A}$ (of vertical type) as above is of the form

$$
\mathcal{A}(X)=\sum \lambda_{\nu}(X)\left(E_{\nu}\right)^{*}
$$

for some uniquely determined maps $\lambda_{\nu}(X): W^{r} P \rightarrow \mathbb{R}$, where $X$ is a rightinvariant vector field on a $\mathcal{P} \mathcal{B}_{m}(G)$-object $P \rightarrow M$. By invariance of $\mathcal{A}$ with respect to $\mathcal{P} \mathcal{B}_{m}(G)$-maps, each $\lambda_{\nu}: T_{G \text {-inv }} \rightsquigarrow T^{(0,0)} W^{r}$ is a $\mathcal{P} \mathcal{B}_{m}(G)$-gaugenatural operator. 


\section{A decomposition}

Proposition 4. Let $\mathcal{A}: T_{G \text {-inv }} \rightsquigarrow T W^{r}$ be a $\mathcal{P} \mathcal{B}_{m}(G)$-gauge-natural operator of order $\leq r$. Then there exists a unique smooth map $\lambda$ : $J_{0}^{r-1}\left(T_{G \text {-inv }}\left(\mathbb{R}^{m} \times G\right)\right) \rightarrow \mathbb{R}$ such that $\mathcal{A}-\mathcal{B}^{\langle\lambda\rangle} \mathcal{W}^{r}$ is of vertical type, where $\mathcal{W}^{r}: T_{G \text {-inv }} \rightsquigarrow T W^{r}$ is the flow operator.

Proof. Let $X$ be a right-invariant vector field on $\mathbb{R}^{m} \times G$.

We can write $\mathcal{A}(X)_{j_{0}^{r}\left(\mathrm{id}_{\mathbb{R}} m_{\times G}\right)}=\mathcal{W}^{r} \widetilde{X}_{j_{0}^{r}\left(\operatorname{id}_{\mathbb{R}} m_{\times G}\right)}$ for some right-invariant vector field $\widetilde{X}$ (see Lemma 2). Suppose $\operatorname{pr}_{\mathbb{R}^{m}} \circ X(0, e) \neq \mu \mathrm{pr}_{\mathbb{R}^{m}} \circ \widetilde{X}(0, e)$ for all $\mu \in \mathbb{R}$ and $\operatorname{pr}_{\mathbb{R}^{m}} \circ \widetilde{X}(0, e) \neq 0$, where $\operatorname{pr}_{\mathbb{R}^{m}}: \mathbb{R}^{m} \times G \rightarrow \mathbb{R}^{m}$ is the projection. Then we have a $\mathcal{P} \mathcal{B}_{m}(G)$-map $\Phi: \mathbb{R}^{m} \times G \rightarrow \mathbb{R}^{m} \times G$ preserving $\theta=j_{0}^{r}\left(\mathrm{id}_{\mathbb{R}^{m} \times G}\right)$ such that

$$
J^{r} T \Phi\left(j_{0}^{r} X\right)=j_{0}^{r} X \quad \text { and } \quad J^{r} T \Phi\left(j_{0}^{r} \widetilde{X}\right) \neq j_{0}^{r} \widetilde{X} .
$$

(Indeed, in some $\mathcal{P} \mathcal{B}_{m}(G)$-trivialization preserving $(0, e)$ we have $X=\partial / \partial x^{1}$ and $\theta=j_{0}^{r} \sigma$. By assumption, there are $i \neq 1$ and $a \neq 0$ such that $\operatorname{pr}_{\mathbb{R}^{m}} \circ \widetilde{X}(0, e)$ $=a \frac{\partial}{\partial x^{i}}(0)+\cdots$, where the dots denote a linear combination of $\frac{\partial}{\partial x^{j}}(0)$ for $j \neq i$. Then we can take $\Phi: \mathbb{R}^{m} \times G \rightarrow \mathbb{R}^{m} \times G$ given by $\Phi\left(x^{1}, \ldots, x^{m}, \eta\right)=$ $\left.\left(x^{1}, \ldots, x^{i}+\left(x^{i}\right)^{r+1}, \ldots, x^{m}, \eta\right).\right)$ Then

$$
\begin{aligned}
\mathcal{A}(X)_{j_{0}^{r}\left(\mathrm{id}_{\mathbb{R}} m_{\times G}\right)} & =\mathcal{W}^{r}\left(\Phi_{*} \widetilde{X}\right)_{j_{0}^{r}\left(\operatorname{id}_{\mathbb{R}^{m}} \times G\right)} \\
& \neq \mathcal{W}^{r}(\widetilde{X})_{j_{0}^{r}\left(\operatorname{id}_{\mathbb{R}^{m}} \times G\right.}=\mathcal{A}(X)_{j_{0}^{r}\left(\operatorname{id}_{\mathbb{R}^{m}} \times G\right)} .
\end{aligned}
$$

This is a contradiction. Consequently, we have

$$
T \pi^{r} \circ \mathcal{A}(X)_{j_{0}^{r}\left(\mathrm{id}_{\mathbb{R}^{m} \times G}\right)}=\lambda\left(j_{0}^{r-1} X\right) \operatorname{pr}_{\mathbb{R}^{m}} \circ X_{(0, e)}
$$

for some (not necessarily unique and not necessarily smooth) map $\lambda$ : $J_{0}^{r-1}\left(T_{G \text {-inv }}\left(\mathbb{R}^{m} \times G\right)\right) \rightarrow \mathbb{R}$ and all right-invariant vector fields on $\mathbb{R}^{m} \times G$ with coefficients (with respect to a fixed basis of right-invariant vector fields on $\mathbb{R}^{m} \times G$ ) being polynomials of degree $\leq r-1$, where $\pi^{r}: W^{r}\left(\mathbb{R}^{m} \times G\right) \rightarrow$ $\mathbb{R}^{m}$ are the projections.

We are going to show that $\lambda$ can be chosen smooth.

Of course (since the left hand side of (1) depends smoothly on $j_{0}^{r} X$ ), the $\operatorname{map} \Phi: J_{0}^{r-1}\left(T_{G \text {-inv }}\left(\mathbb{R}^{m} \times G\right)\right) \rightarrow \mathbb{R}$ given by

$$
\Phi\left(j_{0}^{r-1} X\right)=\lambda\left(j_{0}^{r-1} X\right) X^{1}(0)
$$

is smooth and $\Phi\left(j_{0}^{r-1} X\right)=0$ if $X^{1}(0)=0$, where

$$
X_{(0, e)}=\left.\sum_{i} X^{i}(0) \frac{\partial}{\partial x^{i}}\right|_{0}+(\cdots)
$$

and $(\cdots)$ is the vertical part of $X_{(0, e)}$. Then (as is known from calculus) there is a smooth map $\Psi: J_{0}^{r-1}\left(T_{G \text {-inv }}\left(\mathbb{R}^{m} \times G\right)\right) \rightarrow \mathbb{R}$ such that $\Phi\left(j_{0}^{r-1} X\right)=$ $\Psi\left(j_{0}^{r-1} X\right) X^{1}(0)$. Then we can define new $\lambda=\Psi$. It is equal to the old one if 
$X^{1}(0) \neq 0$. For the new $\lambda$ we have $(1)$ if additionally $X^{1}(0) \neq 0$. Hence we have (1) for all $X$ considered.

Thus $\left(\mathcal{A}(X)-\mathcal{B}^{\langle\lambda\rangle}(X) \mathcal{W}^{r} X\right)_{j_{0}^{r}\left(\mathrm{id}_{\mathbb{R}^{m} \times G}\right)}$ is vertical for all right-invariant vector fields on $\mathbb{R}^{m} \times G$ with coefficients (with respect to a fixed basis of right-invariant vector fields on $\mathbb{R}^{m} \times G$ ) being polynomials of degree $\leq r-1$. Since the union of orbits (with respect to the $\mathcal{P} \mathcal{B}_{m}(G)$-maps preserving $j_{0}^{r}\left(\operatorname{id}_{\mathbb{R}^{m} \times G}\right)$ ) of $j_{0}^{r} X$ for right-invariant $X$ with coefficients (in the above basis) being polynomials of degree $\leq r-1$ is dense in $J_{0}^{r}\left(T_{G \text {-inv }}\left(\mathbb{R}^{m} \times G\right)\right.$ ) (see Lemma 1), $\left(\mathcal{A}(X)-\mathcal{B}^{\langle\lambda\rangle}(X) \mathcal{W}^{r} X\right)_{j_{0}^{r}\left(\mathrm{id}_{\mathbb{R}} m_{\times G}\right)}$ is vertical for all right-invariant vector fields $X$ on $\mathbb{R}^{m} \times G$ with coefficients (in that basis) being polynomials of degree $\leq r$. Thus $\left(\mathcal{A}(X)-\mathcal{B}^{\langle\lambda\rangle}(X) \mathcal{W}^{r}(X)\right)_{j_{0}^{r}\left(\operatorname{id}_{\mathbb{R}^{m}} \times G\right)}$ is vertical for all right-invariant vector fields on $\mathbb{R}^{m} \times G$ (because $\mathcal{A}, \mathcal{W}^{r}$ and $\mathcal{B}$ are of order $\leq r)$. Therefore $\mathcal{A}-\mathcal{B}^{\langle\lambda\rangle} \mathcal{W}^{r}$ is of vertical type by $\mathcal{P} \mathcal{B}_{m}(G)$-invariance and the fact that $W^{r}$ is a transitive bundle functor (i.e. $W^{r} P$ is the $\mathcal{P} \mathcal{B}_{m}(G)$-orbit of $\left.j_{0}^{r}\left(\operatorname{id}_{\mathbb{R}^{m} \times G}\right)\right)$.

5. A classification theorem. We know that any $\mathcal{P} \mathcal{B}_{m}(G)$-gauge-natural operator $\mathcal{A}: T_{G \text {-inv }} \rightsquigarrow T W^{r}$ is of order $\leq r$ (see Proposition 1). Combining Propositions 3 and 4 we get

TheOREM 1. All $\mathcal{P} \mathcal{B}_{m}(G)$-gauge-natural operators $T_{G \text {-inv }} \rightsquigarrow T W^{r}$ form a free $\left(m\left(C_{r}^{m+r}-1\right)+\operatorname{dim}(G) C_{r}^{m+r}+1\right)$-dimensional module over the algebra of maps $J_{0}^{r-1}\left(T_{G \text {-inv }}\left(\mathbb{R}^{m} \times G\right)\right) \rightarrow \mathbb{R}$. The operators $\mathcal{W}^{r}$ and $\left(E_{\nu}\right)^{*}$ for $\nu=$ $1, \ldots, \operatorname{dim}\left(W_{m}^{r} G\right)=m\left(C_{r}^{m+r}-1\right)+\operatorname{dim}(G) C_{r}^{m+r}$ form a basis in this module, where $\left(E_{\nu}\right)$ is a basis of $\mathcal{L}\left(W_{m}^{r} G\right)$ and $E^{*}$ denotes the fundamental vector field on $W^{r} P$ corresponding to $E \in \mathcal{L}\left(W_{m}^{r} G\right)$.

REMARK 1. According to the well-known theory of gauge-natural operators [3], $\mathcal{P B}_{m}(G)$-gauge-natural operators $T_{G \text {-inv }} \rightsquigarrow T W^{r}$ (which are of order $\leq r$ by Proposition 1) are in bijection with $\mathcal{P} \mathcal{B}_{m}(G)$-natural transformations $J^{r} L P \times_{M} W^{r} P \rightarrow T W^{r} P=L W^{r} P \times_{M} W^{r} P$ covering the identity of $W^{r} P$. (For example, the flow operator corresponds to the isomorphism $J^{r} L P \times_{M} W^{r} P=T W^{r} P$ obtained from the Kumpera-Spencer isomorphism $J^{r} L P=L W^{r} P$.) Thus Theorem 1 gives a full description of all $\mathcal{P} \mathcal{B}_{m}(G)$ natural transformations $J^{r} L P \times_{M} W^{r} P \rightarrow T W^{r} P$ covering the identity of $W^{r} P$.

6. Right-invariant gauge-natural operators $T_{G \text {-inv }} \rightsquigarrow T W^{r}$. It is of interest to describe all $\mathcal{P} \mathcal{B}_{m}(G)$-gauge-natural operators $\mathcal{A}: T_{G \text {-inv }} \rightsquigarrow T W^{r}$ such that $\mathcal{A}(X)$ is right $W_{m}^{r} G$-invariant for any right-invariant vector field $X$ on any $\mathcal{P B}_{m}(G)$-object $P \rightarrow M$. For such operators we write $\mathcal{A}: T_{G \text {-inv }} \rightsquigarrow$ $T_{W_{m}^{r} G \text {-inv }} W^{r}$. We cannot extract such operators from the collection described in Theorem 1 . However, we have the following result, which is a consequence 
of the Kumpera-Spencer isomorphism $J^{r} L P=L W^{r} P$ (or more precisely a consequence of Lemma 2).

Proposition 5. There is a bijection between $\mathcal{P} \mathcal{B}_{m}(G)$-gauge-natural operators $\mathcal{A}: T_{G \text {-inv }} \rightsquigarrow T_{W_{m}^{r} G \text {-inv }} W^{r}$ and $\mathcal{P} \mathcal{B}_{m}(G)$-gauge-natural operators $\mathcal{B}: T_{G \text {-inv }} \rightsquigarrow J^{r} T_{G \text {-inv }}$.

Proof. Let $\mathcal{A}: T_{G \text {-inv }} \rightsquigarrow T_{W_{m}^{r} G \text {-inv }} W^{r}$ be a $\mathcal{P} \mathcal{B}_{m}(G)$-gauge-natural operator. Given $X \in \mathcal{X}_{G \text {-inv }}(P)$ and $x \in M$, by Lemma 2 , we have

$$
\mathcal{A}(X)\left|\left(W^{r} P\right)_{x}=\mathcal{W}^{r}(Y(X))\right|\left(W^{r} P\right)_{x}
$$

for some $Y(X) \in \mathcal{X}_{G \text {-inv }}(P)$, and $j_{x}^{r}(Y(X))$ is uniquely determined. We define

$$
\mathcal{B}(X)_{x}:=j_{x}^{r}(Y(X)) .
$$

Clearly $\mathcal{B}: T_{G \text {-inv }} \rightsquigarrow J^{r} T_{G \text {-inv }}$ is a $\mathcal{P} \mathcal{B}_{m}(G)$-gauge-natural operator.

Conversely, let $\mathcal{B}: T_{G \text {-inv }} \rightsquigarrow J^{r} T_{G \text {-inv }}$ be a $\mathcal{P} \mathcal{B}_{m}(G)$-gauge-natural operator. We define a $\mathcal{P} \mathcal{B}_{m}(G)$-gauge-natural operator $\mathcal{A}: T_{G \text {-inv }} \rightsquigarrow T W^{r}$ by

$$
\mathcal{A}_{P}(X)\left|\left(W^{r} P\right)_{x}:=\mathcal{W}^{r} Y\right|\left(W^{r} P\right)_{x},
$$

where $j_{x}^{r} Y=\mathcal{B}(X)_{x}$. Clearly $\mathcal{A}: T_{G \text {-inv }} \rightsquigarrow T_{W_{m}^{r} G \text {-inv }} W^{r}$.

EXAMPLE 4. A simple example of a $\mathcal{P} \mathcal{B}_{m}(G)$-gauge-natural operator $T_{G \text {-inv }} \rightsquigarrow T_{W_{m}^{r} G \text {-inv }} W^{r}$ is $\mathcal{W}^{r}$.

EXAMPLE 5. Another example of a $\mathcal{P} \mathcal{B}_{m}(G)$-gauge-natural operator $T_{G \text {-inv }} \rightsquigarrow T_{W_{m}^{r} G \text {-inv }} W^{r}$ is given by $X \mapsto \mathcal{W}^{r} E^{*}$ for an $\operatorname{Ad}_{G}$-invariant element $E \in \mathcal{L}(G)$. (Then $E^{*} \in \mathcal{X}_{G \text {-inv }}(P)$ and we can apply $\mathcal{W}^{r}$.)

7. An axiomatic characterization of the flow operator $\mathcal{W}^{r}$. Given a $\mathcal{P} \mathcal{B}_{m}(G)$-gauge-natural operator $\mathcal{A}: T_{G \text {-inv }} \rightsquigarrow T W^{r}$ we formulate the following axioms:

Axiom 1. $\mathcal{A}: T_{G \text {-inv }} \rightsquigarrow T_{W_{m}^{r} G \text {-inv }} W^{r}$.

Axiom 2. $\mathcal{A}$ is linear.

Axiom 3. $\mathcal{A}(X)$ is projectable onto $X$ for any $X \in \mathcal{X}_{G \text {-inv }}(P)$.

Clearly the flow operator $\mathcal{W}^{r}: T_{G \text {-inv }} \rightsquigarrow T W^{r}$ satisfies Axioms 1-3.

We have the following complete characterization of $\mathcal{W}^{r}$.

Theorem 2. Let $\mathcal{A}: T_{G \text {-inv }} \rightsquigarrow T W^{r}$ be a $\mathcal{P} \mathcal{B}_{m}(G)$-natural operator satisfying Axioms 1-3. Then $\mathcal{A}=\mathcal{W}^{r}$.

Proof. Let $\mathcal{B}: T_{G \text {-inv }} \rightsquigarrow J^{r} T_{G \text {-inv }}$ correspond to $\mathcal{A}$ in the sense of Proposition 5 . Let $\left(A_{j}\right)$ be a basis of right-invariant vector fields on $G$. By Axioms 
1 and 3 we can write

$$
\mathcal{A}\left(\frac{\partial}{\partial x^{1}}\right)_{0}=j_{0}^{r}\left(\frac{\partial}{\partial x^{1}}+\sum_{i=1}^{m} \sum_{1 \leq|\alpha| \leq r} a_{\alpha}^{i} x^{\alpha} \frac{\partial}{\partial x^{i}}+\sum_{j=1}^{\operatorname{dim}(G)} \sum_{0 \leq|\beta| \leq r} b_{\beta}^{j} x^{\beta} A_{j}\right) .
$$

Then using the invariance of $\mathcal{A}$ with respect to the homotheties $t \mathrm{id}_{\mathbb{R}^{m}} \times \mathrm{id}_{G}$ for $t>0$ and using Axiom 2 we easily see that $\mathcal{A}\left(\partial / \partial x^{1}\right)_{0}=j_{0}^{r}\left(\partial / \partial x^{1}\right)$. Therefore $\mathcal{A}=\mathcal{W}^{r}$ because by the Frobenius theorem, $\mathcal{A}$ is fully determined by its value at $\partial / \partial x^{1}$ over 0 .

8. Some prolongations of connections. Using the flow operator $\mathcal{W}^{r}$ one can lift completely pairs $(\Gamma, \Lambda)$ consisting of principal connections $\Gamma$ on $P \rightarrow M$ and linear connections $\Lambda: T M \rightarrow J^{r} T M$ on $M$ to principal connections $\mathcal{W}^{r}(\Gamma, \Lambda)$ on $W^{r} P \rightarrow M$ as follows.

EXAMPLE 6 (see [3]). An rth order linear connection on $M$ is a linear splitting $\Lambda: T M \rightarrow J^{r} T M$. Consider a vector field $X$ on $M$ and denote by $\Gamma X$ its $\Gamma$-lift with respect to a principal connection $\Gamma$ on $P \rightarrow M$. The flow prolongation $\mathcal{W}^{r}(\Gamma X)$ is a vector field on $W^{r} P$ depending on $r$-jets only, which can be interpreted as a bundle map $W^{r} P \times_{M} J^{r} T M \rightarrow T W^{r} P$. Then the composition of the map $W^{r} P \times_{M} J^{r} T M \rightarrow T W^{r} P$ with the restriction of $\operatorname{id}_{W^{r} P} \times \Lambda$ to $W^{r} P \times_{M} T M$ is a lifting map $W^{r} P \times_{M} T M \rightarrow T W^{r} P$ of a principal connection $\mathcal{W}^{r}(\Gamma, \Lambda)$ on $W^{r} P \rightarrow M$.

The right action of $W_{m}^{r} G$ on $W^{r} P$ induces (by restriction) a right action of the subgroup $G_{m}^{r} \rtimes\left(T_{m}^{r} G\right)_{e} \subset W_{m}^{r} G$ on $W^{r} P$, where $\left(T_{m}^{r} G\right)_{e}$ is the fibre of $T_{m}^{r} G$ over $e$. This induced right action preserves the obvious projection $W^{r} P \rightarrow P, j_{0}^{r} \Phi \mapsto \Phi(0, e)$. Then $W^{r} P \rightarrow P$ is a principal bundle with the standard Lie group $G_{m}^{r} \rtimes\left(T_{m}^{r} G\right)_{e}$. The following question arises. Given an $r$ th order linear connection $\Lambda$ on $M$ and a principal connection $\Gamma$ on a principal $G$-bundle $P \rightarrow M$, can one construct a principal connection $\widetilde{\mathcal{W}}^{r}(\Gamma, \Lambda)$ on $W^{r} P \rightarrow P$ ? In view of the following example the answer is affirmative for abelian Lie groups $G$.

ExAMPLE 7. Let $G$ be an abelian Lie group. Let $\Gamma$ be a principal connection on $\pi: P \rightarrow M$ and $\Lambda: T M \rightarrow J^{r} T M$ be an $r$ th order linear connection on $M$. We define a lifting map $\widetilde{\mathcal{W}}^{r}(\Gamma, \Lambda): W^{r} P \times_{P} T P \rightarrow T W^{r} P$ as follows: Let $(w, X) \in\left(W^{r} P \times_{P} T P\right)_{p}, p \in P_{x}, x \in M$. The connection $\Gamma$ yields the decomposition $X=\widetilde{X}+A$ into the $\Gamma$-horizontal and vertical parts. Let $A^{*}$ be the unique fundamental vector field on $P \rightarrow M$ passing through $A$. Let $Y \in T_{p} P$ be the projection of $\mathcal{W}^{r}(\Gamma, \Lambda)(w, T \pi(\tilde{X}))$, where $\mathcal{W}^{r}$ is as in Example 6 . Let $Y=\widetilde{X}+B$ be the decomposition of $Y$ into the $\Gamma$-horizontal and vertical parts. Let $B^{*}$ be the unique fundamental vector field on $P \rightarrow M$ passing through $B$. Since $G$ is abelian, $A^{*}$ and $B^{*}$ are right-invariant vector 
fields on $P \rightarrow M$, and we can apply the flow operator $\mathcal{W}^{r}$ to $A^{*}$ and $B^{*}$. We put

$$
\widetilde{\mathcal{W}}^{r}(\Gamma, \Lambda)(w, X):=\mathcal{W}^{r}(\Gamma, \Lambda)(w, T \pi(\widetilde{X}))+\mathcal{W}^{r} A^{*}(w)-\mathcal{W}^{r} B^{*}(w) .
$$

Clearly, $\widetilde{\mathcal{W}}^{r}(\Gamma, \Lambda)$ defines a principal connection on $W^{r} P \rightarrow P$.

REMARK 2. Let $\mathcal{F} \mathcal{M}_{m, n}$ be the category of fibred manifolds with $n$ dimensional fibres and $m$-dimensional bases and their fibred embeddings. The construction from Example 6 is a direct modification of a quite similar construction for $r$ th order bundle functors $F: \mathcal{F} \mathcal{M}_{m, n} \rightarrow \mathcal{F M}$ (see [3]). In [6], we proved that if a bundle functor $F: \mathcal{F} \mathcal{M}_{m, n} \rightarrow \mathcal{F} \mathcal{M}$ admits an $\mathcal{F} \mathcal{M}_{m, n}$-natural operator $C$ transforming a general connection $\Gamma$ on $p$ : $Y \rightarrow M$ and a linear connection $\Lambda$ on $M$ into a general connection $C(\Gamma, \Lambda)$ on $F Y \rightarrow Y$, then $F Y=p^{*} G M$ for some bundle functor (natural bundle) $G: \mathcal{M} f_{m} \rightarrow \mathcal{F} \mathcal{M}$ on the category $\mathcal{M} f_{m}$ of $m$-dimensional manifolds and embeddings. Thus Example 7 shows that a result similar to [6] is not true for gauge bundle functors $F: \mathcal{P} \mathcal{B}_{m}(G) \rightarrow \mathcal{F} \mathcal{M}$ with abelian Lie groups $G$.

\section{References}

[1] J. Gancarzewicz, Liftings of functions and vector fields to natural bundles, Dissertationes Math. 212 (1983).

[2] I. Kolář, Connections on principal prolongations of principal bundles, in: Differential Geometry and its Applications, Proc. Conf. in Honour of Leonard Euler (Olomouc, 2007), World Sci., 2008, 279-291.

[3] I. Kolář, P. W. Michor and J. Slovák, Natural Operations in Differential Geometry, Springer, 1993.

[4] A. Kumpera and D. Spencer, Lie Equations I, Princeton Univ. Press, 1972.

[5] J. Kurek and W. M. Mikulski, Lifting vector fields to the rth order frame bundle, Colloq. Math. 111 (2008), 51-58.

[6] W. M. Mikulski, On the existence of some type constructions of connections, Reports Math. Phys. 58 (2006), 411-416.

[7] A. Morimoto, Prolongations of connections to bundles of infinitely near points, J. Differential Geom. 11 (1976), 479-498.

Institute of Mathematics

Jagiellonian University

Łojasiewicza 6

30-348 Kraków, Poland

E-mail: Wlodzimierz.Mikulski@im.uj.edu.pl 